\title{
EFFECTS OF NITROGEN AND PHOSPHORUS ON PYROLYTIC GAS FORMATION FROM CELLULOSIC FIBERS
}

\author{
Shigeko Nakanishi, Fumiko Ohkouchi, Fumi Masuko, and Ikuko Nishimoto* \\ Department of Clothing, Faculty of Home Economics, Japan Women's University, \\ 2-8-1. Mejirodai, Bunkyo-ku. Tokyo, 112 Japan
}

\begin{abstract}
Flame retardant effects of nitrogen and / or phosphorous treatments of cotton fabrics were in. vestigated through the analysis of pyrolytic gas formation. While the pyrolytic behavior of $N$ - and/or $P$ treated samples with low limiting oxygen index (LOI) was similar to that of the untreated material, the behavior of samples with high LOI was remarkably altered. In particular, the sample treated with $20 \%$ diammonium hydrogen phosphate showed a large decrease in the generation of a poisonous gas (acrolein) and flammable gases, and showed an increase in the formation of unsaturated cyclic compounds, which contrib. uted to the self-extinguishing behavior. These results were attributed to the synergistic effect of nitrogen and phosphorus compounds added to the cellulosic materials.
\end{abstract}

\section{INTRODUCTION}

For preventing the generation of hazardous gases in fire incidents, it is important to investigate the behavior of pyrolytic gas formation from flammable polymers. Thermal degradation of cellulosic fibers was investigated by Inagaki et al. [1-4] and other groups [5-9]. However, the effect of the individual flame retardant and the synergistic effect on pyrolytic gas formation have not been fully elucidated. Nakanishi [10] studied the effects of flame retandant finishings for cellulosic fibers in terms of the generation of $\mathrm{CO}$. $\mathrm{CO}_{2}$ and smoke. However, the information is still limited on the effects of flame retandant elements.

In this study, we attempted a quantitative analysis of pyrolytic gases from flame retardant-finished cellu. losic fibers, with special emphasis on the effects of $\mathrm{N}$ and / or P as flame retardant elements.

\section{EXPERIMENTAL}

\subsection{Materials}

\subsubsection{Substrate}

Cotton $100 \%$ fabric was purified by Soxhlet extrac tion with chloroform-methanol $(1: 1 \mathrm{vol} / \mathrm{vol})$ for $24 \mathrm{~h}$.

\footnotetext{
* Present Address: Medical School, Okayama University. 3-3-1, Tsushimanaka, Okayama City. 700 Japan
}

\subsubsection{Reagents}

Compounds used for treatments are shown in Table

1. Ammonium hydroxide $\left(\mathrm{NH}_{4} \mathrm{OH}\right)$ and urea were used

Table 1 Limiting Oxygen Index (LOI) of Untreated and Treated Cotton Samples

\begin{tabular}{cr}
\hline Reagent & LOI $(\%)$ \\
\hline Untreated & 18.9 \\
$20 \%$ urea & 22.2 \\
$20 \% \mathrm{NH}_{4} \mathrm{OH}$ & 19.0 \\
$5 \% \mathrm{H}_{3} \mathrm{PO}_{4}$ & 28.1 \\
$20 \% \mathrm{H}_{3} \mathrm{PO}_{4}$ & 46.1 \\
$20 \% \mathrm{Na}_{2} \mathrm{HPO}_{4}$ & 22.2 \\
$20 \%$ urea $+\mathrm{Na}_{2} \mathrm{HPO}_{4}$ & 23.5 \\
$5 \%$ urea $+\mathrm{H}_{3} \mathrm{PO}_{4}$ & 24.8 \\
$20 \%$ urea $+\mathrm{H}_{3} \mathrm{PO}_{4}$ & 46.1 \\
$20 \% \mathrm{NH}_{4} \mathrm{OH}_{+}+\mathrm{H}_{3} \mathrm{PO}_{4}$ & 43.1 \\
$5 \%\left(\mathrm{NH}_{4}\right)_{2} \mathrm{HPO}_{4}$ & 24.4 \\
$20 \%\left(\mathrm{NH}_{4}\right)_{2} \mathrm{HPO}_{4}$ & 43.4 \\
\hline
\end{tabular}

as nitrogen sources, phosphoric acid $\left(\mathrm{H}_{3} \mathrm{PO}_{4}\right)$ and disodium hydrogen phosphate $\left(\mathrm{Na}_{2} \mathrm{HPO}_{4}\right)$ as phosphorus sources, and diammonium hydrogen phosphate $\left[\left(\mathrm{NH}_{4}\right)_{2} \mathrm{HPO}_{4}\right]$ as a compound containing both ni. trogen and phosphorus in the same molecule. $N$-source and $P$-source compounds were combined for studying the effect of the combination of $\mathrm{N}$ and $\mathrm{P}$ for compari son with $\left(\mathrm{NH}_{4}\right)_{2} \mathrm{HPO}_{4}$. 


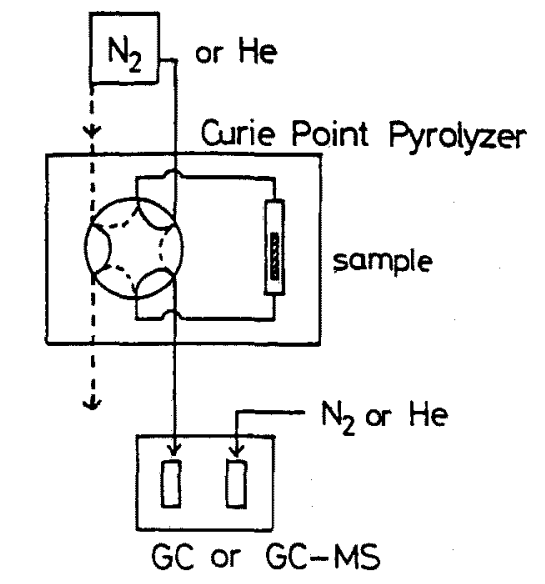

... purging with $\mathrm{N}_{2}$ gas

- to GC column

Fig. 1. A schematic illustration of $P G C$ and PGC-MS apparatus.

\subsection{Methods}

\subsubsection{Treatment}

Five percent and $20 \%$ solutions of the above mentioned reagents were prepared, in which the purified cotton fabric was immersed for $5 \mathrm{~min}$. The sample was mangled and dried indoors after a 2 dip- 2 nip process. The pick up was adjusted to about $90 \%$.

\subsubsection{Pyrolysis Gas Chromatography (PGC)}

\section{(1) Apparatus}

A high-frequency induction-heating-furnace pyrolyzer, Type JHP-2 (Nihon Bunseki Kogyo) was connected with a gas chromatograph (GC) Type 063 (Hitachi Seisakusho) with a FID detector to pyrolyze and analyze pyrolytic gases simultaneously (see Fig. 1). The data were treated with Chromatopack C-R1B (Shimadzu Seisakusho).

(2) Conditions for analyses

(i) Pyrolysis: Three kinds of Pyrofoiles (foils made of ferromagnetic derivatives, Nihon Kagaku Kogyo) for 445,590 and $764^{\circ} \mathrm{C}$ were selected for representative pyrolysis. A $200-300 \mu \mathrm{g}$ portion of a sample, precisely weighed, was wrapped with a foil and pyroly. zed by high frequency heating. The furnace temperature was $160^{\circ} \mathrm{C}$ and the pipe temperature was 210 ${ }^{\circ} \mathrm{C}$.

(ii) GC analysis: Porapak $Q$ of $80-100$ mesh was packed in a glass column, $3 \mathrm{~mm}$ in diameter. GC analysis was carried out under the following conditions: The temperature was raised from $50^{\circ} \mathrm{C}$ (cooled down with dry ice) to $200^{\circ} \mathrm{C}$ at a rate of $5{ }^{\circ} \mathrm{C} / \mathrm{min}$ under a nitrogen flow of $40-60 \mathrm{~mL} / \mathrm{min}$. The injection inlet was kept at $200^{\circ} \mathrm{C}$

\subsubsection{Pyrolysis Gas Chromatography - Mass Spectrometry (PGC - MS)}

\section{(1) Apparatus}

The pyrolyzer mentioned above was connected to a gas chromatograph-mass spectrometer, Type JMS-DX 300 (Nihon Denshi) for the identification of gaseous compounds. The data were analyzed with JMA 3000 (Nihon Denshi).

(2) Conditions for analysis

(i) Pyrolysis: Pyrolysis was carried out under the conditions described in 2.2.2, (2), (i).

(ii) PGC-MS analysis: Porapak Q, same as that used in PGC analysis was packed in a glass column. Electron ionization (EI) and chemical ionization (CI) methods were adopted for analysis at an ionization voltage of $70 \mathrm{eV}$. PGC-MS analysis was carried out from $50^{\circ} \mathrm{C}$ to $240^{\circ} \mathrm{C}$ at a programmed rate of $4{ }^{\circ} \mathrm{C} / \mathrm{min}$. The repetition was made by a 6 -second cycle in a scan range of $15-300 \mathrm{~m} / \mathrm{z}$. The temperatures of the separator and the ionization chamber were set at $260^{\circ} \mathrm{C}$ and $200{ }^{\circ} \mathrm{C}$, respectively. The ion acceleration voltage was $3.5 \mathrm{kV}$ and the flow rate of $\mathrm{He}$ was adjusted to $40 \mathrm{~mL} / \mathrm{min}$. Repetition ion current chromatograms were used for the identification of gases. The structure of each pyrolytic gaseous product was identified from pseudo-molecular ions and mass fragments. The identification was also confirmed with authentic compounds.

Pyrolysis and chromatographic analysis were repeated 5-10 times for each sample and the results were averaged. The amount of each pyrolytic compound was shown as an amount per $1 \mathrm{~g}$ of the substrate (cotton) in each treated sample after determining its addon.

\subsubsection{Evaluation of Flame Retardancy}

As a measure of flame retardancy, we adopted the limiting oxygen index (LOI) which is defined as the value of minimum volume $\%$ of oxygen in $\mathrm{O}_{2}-\mathrm{N}_{2}$ mix. ture required for the combustion of materials under the regulated testing conditions. LOI values were de- 

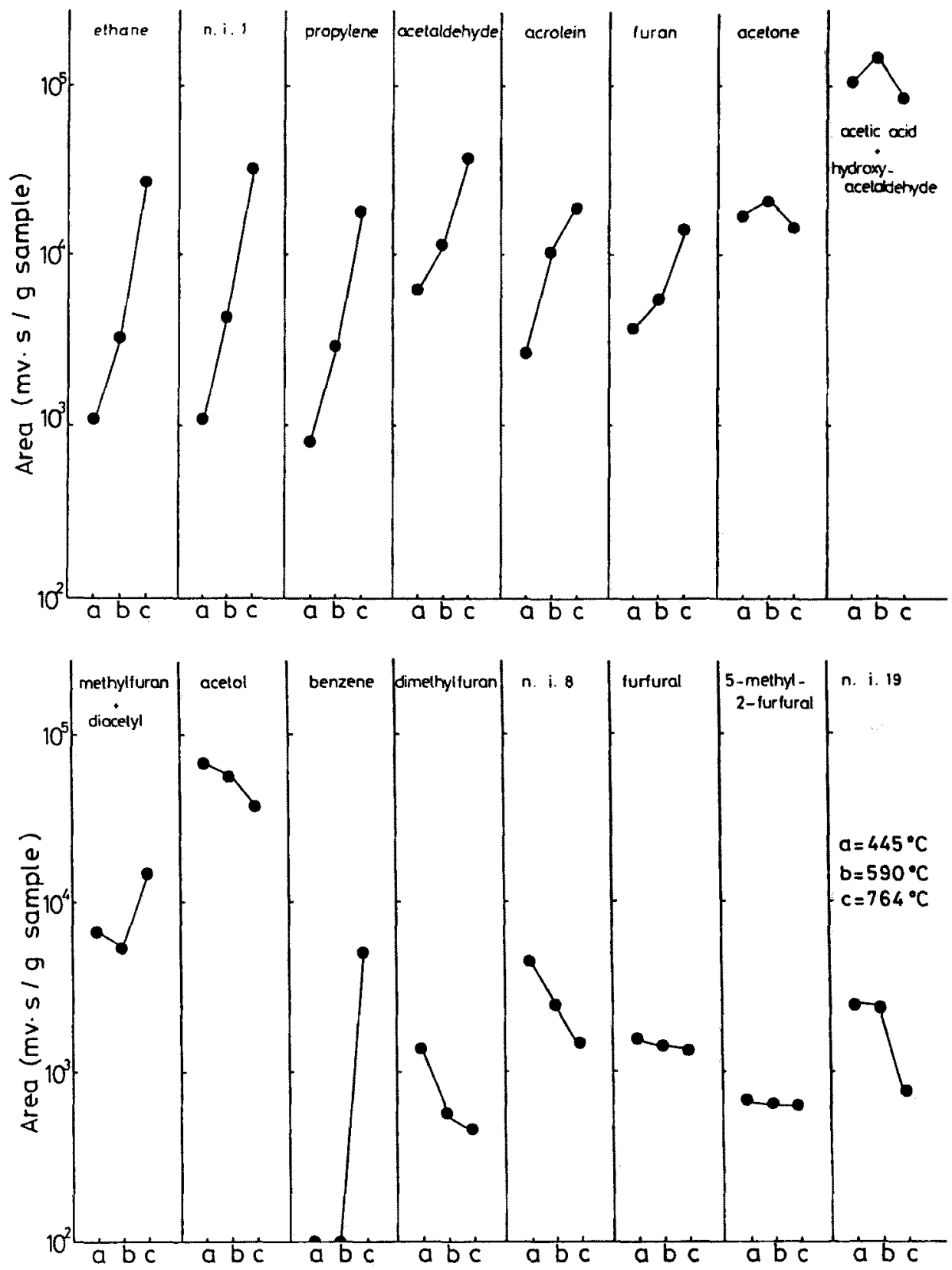

Fig. 2. Temperature dependence of the formation of gaseous products from cotton fibers. Pyrolyzed at (a), $445^{\circ} \mathrm{C} ;(\mathrm{b})$, $590^{\circ} \mathrm{C} ;$ (c). $764^{\circ} \mathrm{C}$.

termined based on JIS K $7201-1976$ by using a burning tester ON-1 (Suga Shikenki).

\section{RESULTS AND DISCUSSION}

The thermal treatment was carried out in $\mathrm{N}_{2}$ flow by the instaneous pyrolysis which reaches the fixed temperature within $0.2 \mathrm{~s}$. This is to avoid or minimize the influence of oxidation and secondary decompositions. 


\subsection{Temperature Dependence of Pyrolytic Gas Formation}

The purpose of this experiment was to determine the optimal heating conditions with reliability for the present study. An attempt was made to observe pyrolytic gas formation in three temperature ranges, i.e., $400-500^{\circ} \mathrm{C}$, around $600^{\circ} \mathrm{C}$ and $700-800^{\circ} \mathrm{C}$.

The result of GC analysis clearly showed a temperature dependence of pyrolytic gas formation as illustrated in Fig. 2. The amounts of hydrocarbons with low boiling point increased with a rise in temperature and showed a remarkable increase in a temperature range higher than $700{ }^{\circ} \mathrm{C}$. This tendency is similar to that shown by Hofmann and Raschorof (5). On the contrary, the amounts of acetol, dimethylfuran, furfural, 5-methyl-2-furfural, and peaks n.i. (not identified) No. 8 and No. 19 decreased with a rise in temperature. No. 19 gas was characterized by fragment ions of $m / z=29.57$ and 69 , and by molecular weight of 114 which is the highest among those of the pyrolytic gaseous compounds detected in this study. The same pyrolytic product was observed from cellulose by Wedley (6) as a not-identified product.

The comparison of furan (without $-\mathrm{CH}_{3}$ group), methylfuran (with one $-\mathrm{CH}_{3}$ group) and dimethylfuran (with two $-\mathrm{CH}_{3}$ groups) showed that the amounts of furan and methylfuran increased while that of dimethylfuran decreased as the temperature rose. Such a tendency means that the scission of $-\mathrm{CH}_{3}$ group from dimethylfuran occurred to form methyl. furan and / or furan. The decrease in the amount of furfural suggests another route to furan by removal of $-\mathrm{CHO}$ group from furfural.

The amounts of compounds with boiling point from $60^{\circ} \mathrm{C}$ to around $115^{\circ} \mathrm{C}$, i.e., acetone, hydroxy acetaldehyde and acetic acid. showed maxima at around $600{ }^{\circ} \mathrm{C}$ and decreased at $700{ }^{\circ} \mathrm{C}$ or higher. The amount of benzene increased markedly, as much as 50 times, above $700^{\circ} \mathrm{C}$. This suggests that the formation of benzene is attributed to the double-bond formation between $\mathrm{C}$ atoms through dehydration of cellulose in thermal decomposition.

A bove findings show that the thermal decomposition was insufficient in a range of $400-500{ }^{\circ} \mathrm{C}$ and too much proceeded in a range of $700-800^{\circ} \mathrm{C}$. Therefore, the pyrolysis temperature of about $600^{\circ} \mathrm{C}$ is the most appropriate for the purpose of this study.

\subsection{Effects of $\mathbf{N}$ and $\mathbf{P}$ on Pyrolytic Gas Formation}

\subsubsection{Effects on LOI}

The LOI value was chosen as an indicator of flame retardancy to examine the relationship between flame retardancy and the pyrolytic gas formation. A high LOI value means that the material requires higher concentration of oxygen to keep burning, and the LOI of 27-28 is regarded as the critical level for the material to be flammable.

Table 1 shows that samples treated with compounds containing $\mathrm{N}$ or $\mathrm{P}$ alone except those treated with $\mathrm{H}_{3} \mathrm{PO}_{4}$ had LOI values lower than the critical level, $27-28 \%$. High LOI values of $\mathrm{H}_{3} \mathrm{PO}_{4}$-containing samples can be attributed not only to $\mathrm{P}$ element but also to high moisture content owing to hygroscopicity of $\mathrm{H}_{3} \mathrm{PO}_{4}$. High flame-retarding effect was also observed for the sample treated with $20 \%\left(\mathrm{NH}_{4}\right)_{2}$ $\mathrm{HPO}_{4}$, probably because of the synergism of $\mathrm{N}$ and $\mathrm{P}$. However, those treated with a mixture of urea and $\mathrm{Na}_{2} \mathrm{HPO}_{4}$ showed little flame-retarding effect though they contain both $\mathrm{N}$ and $\mathrm{P}$. This fact suggests that the contribution of $\mathrm{N}$ and $\mathrm{P}$ together to flame retardancy requires a proper mutual pasition of the elements to show the synergism for flame retardation. It was also found that the insufficient add-on of flame retardants failed to give flame retardancy.

Taking the above results into consideration, attention was paid to the relationship between LOI values and pyrolytic gas formation. PG chromatograms of untreated and treated samples obtained by PGC-MS analysis showed approximately 46 peaks of pyrolytic gaseous compounds. Of these, we paid attention to 19 compounds definitely identified and two unidentified compounds, No. 8 and No. 18, which showed markedly different behavior in the formation between flame retardant samples and samples with low LOI values.

Fig. 3 shows relative amounts of these pyrolytic gaseous compounds from various samples. The amount from the untreated sample is indicated by a broken line for each product. In this figure, the following different behavior is observed between the untreated sample and the samples containing $\mathrm{N}$ and/or $P$, and also between samples with high LOI values and those with low LOI values. 

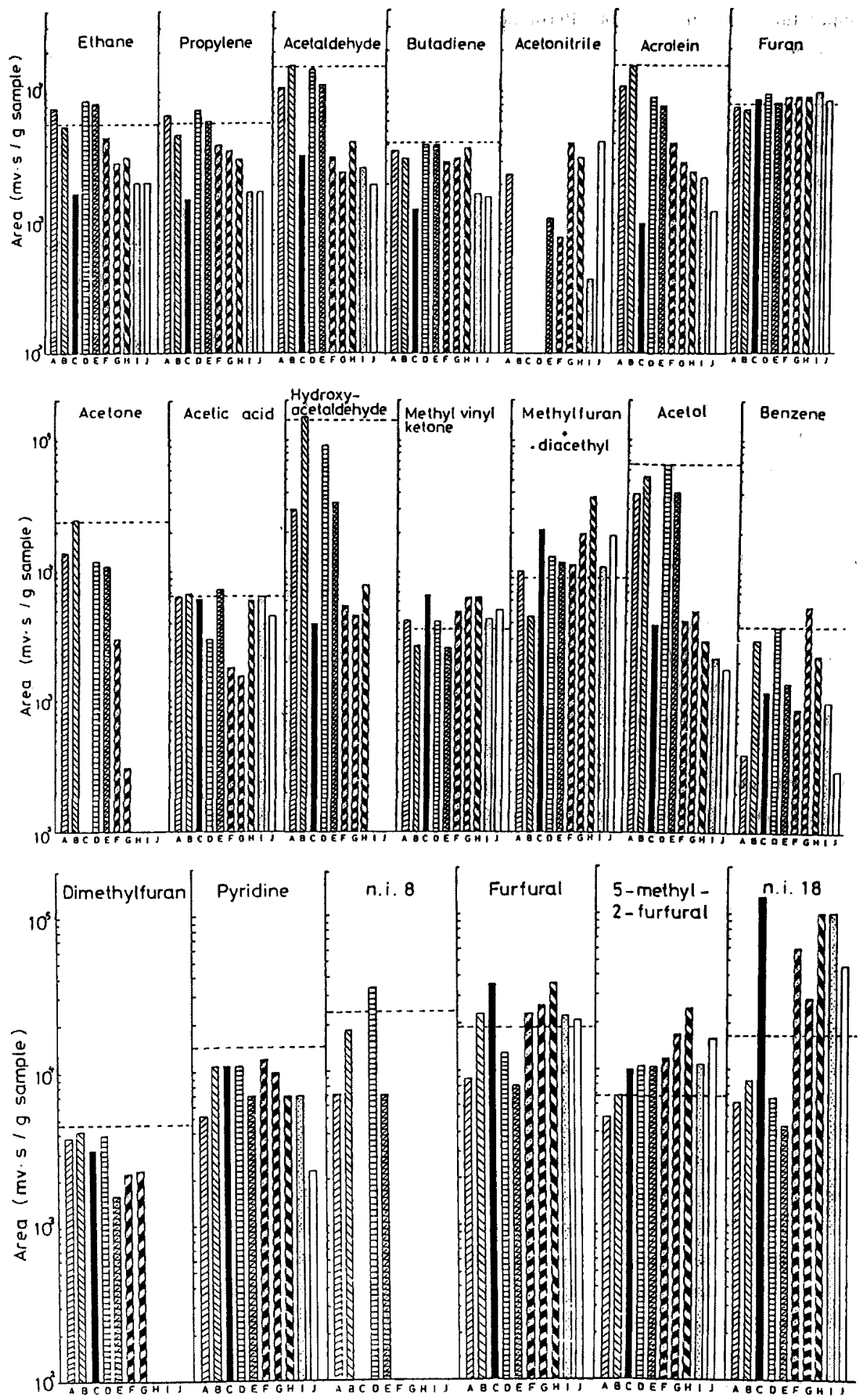

Fig. 3. Gaseous products from untreated and treated cotton samples. $\cdots \cdots$, Untreated. Treated samples with: (A). $20 \%$ urea; (B), $20 \% \mathrm{NH}_{4} \mathrm{OH}$; (C), $20 \% \mathrm{H}_{3} \mathrm{PO}_{4}$; (D), $20 \% \mathrm{Na}_{2} \mathrm{HPO}_{4} ;$ (E), $20 \%$ urea $+\mathrm{Na}_{2} \mathrm{HPO}_{4} ;$ (F), $5 \%$ urea $+\mathrm{H}_{3} \mathrm{PO}_{4} ;(\mathrm{G}), 20 \%$ urea $+\mathrm{H}_{3} \mathrm{PO}_{4} ;(\mathrm{H}), 20 \% \mathrm{NH}_{4} \mathrm{OH}+\mathrm{H}_{3} \mathrm{PO}_{4} ;(\mathrm{I}) .5 \%\left(\mathrm{NH}_{4}\right)_{2} \mathrm{HPO}_{4}$ and $(\mathrm{J}) .20 \%\left(\mathrm{NH}_{4}\right)_{2} \mathrm{HPO}_{4}$. 


\subsubsection{Reduction of Flammable Gas Formation}

The amounts of propylene, acetaldehyde, butadiene, acrolein, acetone, hydroxyacetaldehyde, acetol, benzene, dimethylfuran, ethane and n.i. No. 8 from flame retardant samples were considererably less than those from flammable samples with low LOI values (see Table 1), e.g., acetaldehyde and benzene decreased to $1 / 8-1 / 13$; acetol to $1 / 30-1 / 40$; and, acetone, hydroxyacetaldehyde, dimethylfuran and n.i. No. 8 entirely disappeared:

\subsubsection{Inhibition of Acrolein Generation}

Samples without flame retardancy such as those treated with urea or $\mathrm{NH}_{4} \mathrm{OH}$, containing $\mathrm{N}$ alone, showed little change in the generation of acrolein. Those treated with $\mathrm{Na}_{2} \mathrm{HPO}_{4}$ alone or urea $+\mathrm{Na}_{2}$ $\mathrm{HPO}_{4}$ showed only a small decrease. On the other hand, flame retardant samples treated with $\left(\mathrm{NH}_{4}\right)_{2}$ $\mathrm{HPO}_{4}, \mathrm{H}_{3} \mathrm{PO}_{4}$, urea $+\mathrm{H}_{3} \mathrm{PO}_{4}$ and $\mathrm{NH}_{4} \mathrm{OH}+\mathrm{H}_{3} \mathrm{PO}_{4}$ etc. showed much larger reduction. Byrne et al. (7) suggested that the presence of $P$ and / or $N$ might inhibit the formation of acrolein.

\subsubsection{Increase in the Amount of Unsaturated Compounds}

Methyl vinyl ketone, furan and its derivatives, i.e., methylfuran, furfural and 5-methyl-2-furfural containing carbon-carbon double bonds produced from the treated samples showed an increase in amount as compared with those from the untreated sample. A larger increase was observed for the samples containing $\mathrm{N}$ and $\mathrm{P}$ with higher LOI values. These products are likely to be related to char formation, because the findings well correspond to the observation of IR absorption spectra of residual condensed phase obtained by the thermal treatment of cellulose-ammonium phosphate $(8)$.

Since peaks of methylfuran and diacetyl could not be resolved from each other, combined values are indicated in Fig. 3 . However, the amounts of methylfuran can be calculated from the relative molar ratios of methylfuran and diacetyl based on the intensity ratios in the fragmentation patterns obtained by PGC-MS analysis. The molar ratio of methylfuran is around $10 \%$ for the untreated sample, $24 \%$ for samples containing $\mathrm{P}$ alone, $38 \%$ for those containing $\mathrm{N}$ alone and up to $66 \%$ for those containing both $\mathrm{N}$ and $\mathrm{P}$. The result by Hofmann and Raschorof [5] also showed a similar tendency, i.e., finished samples generated methylfuran twice as much as an unfinished sample.

\subsubsection{Acetonitrile Generation}

Acetonitrile is an extremely useful substance for flame retardation as a radical scavenger in the vapor phase. Acetonitrile was detected neither from untreated sample nor from those treated with compounds containing $\mathrm{N}$ or $\mathrm{P}$ alone, but was generated from samples containing both $\mathrm{N}$ and $\mathrm{P}$. It is notable that samples with high LOI values treated with $20 \%\left(\mathrm{NH}_{4}\right)_{2}$ $\mathrm{HPO}_{4}, \mathrm{NH}_{4} \mathrm{OH}+\mathrm{H}_{3} \mathrm{PO}_{4}$ and urea $+\mathrm{H}_{3} \mathrm{PO}_{4}$ gave the largest amounts, while samples with low LOI values because of the low level of add-on yielded much less amounts of acetonitrile even though they contain both $\mathrm{N}$ and $\mathrm{P}$. This fact confirms a close relationship between the formation of acetonitrile and the flame re. tardancy.

According to Garn and Denson [9], acetonitrile is formed from acetic acid, a pyrolytic product from cellulose, and ammonia generated from flame retardants, e.g., ammonium salts, with the aid of catalytic action of P. Acetonitrile is converted into nitrogen oxides which play a role to give the self-extinguishability by acting as an inhibitor for radical side chain reactions. In contrast to our results, Hofmann and Raschorof [5] reported the production of very toxic and explosive acrylonitrile from fabric finished with Pyrovatex $(N$-methyloldialkyl-phosphonopropionamide, produced by Albright \& Wilson Co.). This difference is probably due to the difference between ammonium salts and the organic phosphonocarbamate containing $-\mathrm{CH}_{2}$ $\mathrm{CH}_{2} \mathrm{CONHCH}_{2} \mathrm{OH}$. Acrylonitrile was also detected in

(A.B)

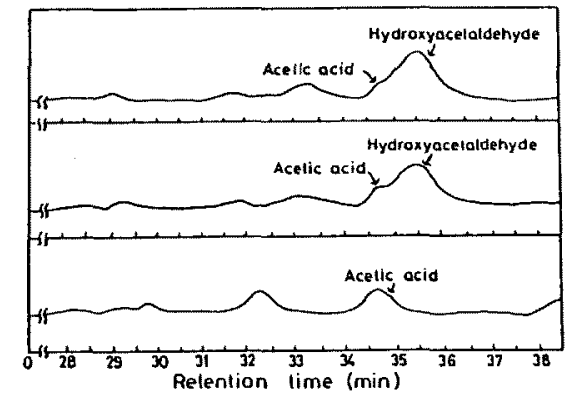

Fig. 4. Mutual change in amounts of acetic acid and hydroxyacetaldehyde pyrogramatically observed in untreated and treated cotton samples. (A), Untreated; (B). treated with $\mathrm{Na}_{2} \mathrm{HPO}_{4}$ and (D), treated with $\left(\mathrm{NH}_{4}\right)_{2} \mathrm{HPO}_{4}$. 
our investigation for a sample treated with Pyrovatex.

The quantitative change in the amount of acetic acid is important in connection with the formation of acetonitrile. Acetic acid was detected as a shoulder of the peak of hydroxyacetaldehyde for the untreated sample and samples treated with compounds contain. ing $N$ or $P$ alone. On the other hand, hydroxyacetaldehyde disappeared and only acetic acid became detectable for samples treated with $\left(\mathrm{NH}_{4}\right)_{2} \mathrm{HPO}_{4}$ (Fig. 4).

According to Byrne et al., [7], hydroxyacetaldehyde is formed from 1,2-ethenediol, an intermediate pyrolytic product generated by the spliting of a glucose unit through the following process:

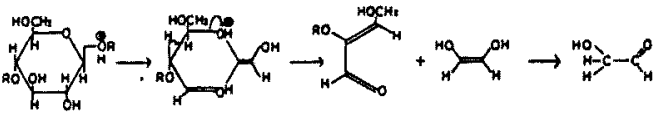

The pyrolysis of the untreated sample or samples containing $\mathrm{N}$ or $\mathrm{P}$ alone will proceed mainly through this route.

On the other hand, it has been considered that acetic acid is formed from ketone, a dehydration product of hydroxyacetaldehyde, by the addition of water generated during the pyrolysis of cellulose. When samples treated with $\left(\mathrm{NH}_{4}\right)_{2} \mathrm{HPO}_{4}$, containing $\mathrm{N}$ and $\mathrm{P}$ together, are pyrolyzed, this process seems to proceed mainly to form acetic acid.

The compound n.i. No. 18 is also worth paying attention since it was detected in a very large amount only from samples containing $\left(\mathrm{NH}_{4}\right)_{2} \mathrm{HPO}_{4}$ and / or $\mathrm{H}_{3} \mathrm{PO}_{4}$. This product showed fragment ions of $\mathrm{m} / z=$ 39,53 and 68 , typical for furan. The molecular weight detemined by $\mathrm{CI}-\mathrm{MS}$ was 96 which is the same as that of furfural.

\subsubsection{Comparison of Total Amounts of Pyrolytic Gases}

Fig. 5 shows the total amounts of pyrolytic gases from the samples. The untreated sample gave the largest amount and those treated with $\mathrm{NH}_{4} \mathrm{OH}(\mathrm{B})$ and $\mathrm{Na}_{2} \mathrm{HPO}_{4}$ (D) containing $\mathrm{N}$ or $\mathrm{P}$ alone showed slightly lower level of gas formation. Such findings are consist ent with their low LOI values, while samples contain ing urea $(A, E, F, G)$ showed less amounts than $B$ or $D$ though they contain $\mathrm{N}$ alone and LOI values are low just as samples B and D. All samples with high LOI values gave much less amounts of pyrolytic gases.

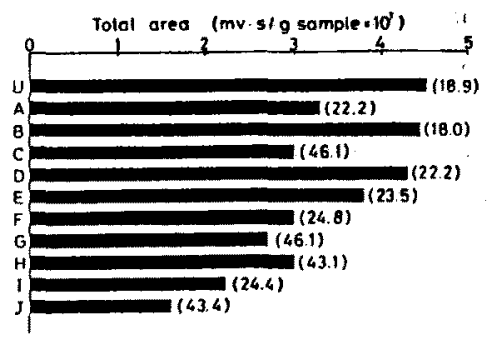

Fig. 5. Total amount of pyrolytic gases. (U), Untreated. Treated samples with: (A), $20 \%$ urea; (B), $20 \% \mathrm{NH}_{4} \mathrm{OH}$; (C). $20 \% \mathrm{H}_{3} \mathrm{PO}_{4}$; (D), $20 \% \mathrm{Na}_{2} \mathrm{HPO}_{4}$ (E), $20 \%$ urea $+\mathrm{Na}_{2}$ $\mathrm{HPO}_{4} ;(\mathrm{F}) .5 \%$ urea $+\mathrm{H}_{3} \mathrm{PO}_{4} ;(\mathrm{G}), 20 \%$ urea $+\mathrm{H}_{3} \mathrm{PO}_{4}$; (H). $20 \% \quad \mathrm{NH}_{4} \mathrm{OH}+\mathrm{H}_{3} \mathrm{PO}_{4}$; (I). $5 \%\left(\mathrm{NH}_{4}\right)_{2} \mathrm{HPO}_{4}$ and (J), $20 \%\left(\mathrm{NH}_{4}\right)_{2} \mathrm{HPO}_{4}$. Numbers in parentheses indicate LOI values.

Sample J treated with $20 \%\left(\mathrm{NH}_{4}\right)_{2} \mathrm{HPO}_{4}$ showed the least amount exhibiting a synergistic effect of $\mathrm{N}$ and $P$ present in the same molecule.

It is concluded from the above results that the flame retarding effects, particularly the synergistic effects of $N$ and $P$, are well reflected in the pyrolytic behavior, and showed a good agreement with the inhibition of flammable gas formation.

\section{REFERENCES}

1. N. Inagaki and K. Katsuura, Kogyo Kagaku Zasshi, 72, 2303 (1969)

2. N. Inagaki and K. Katsuura, Kogyo Kagaku Zasshi, 73, 1433 (1970)

3. N. Inagaki and K. Katsuura, Kogyo Kagaku Zasshi, 74, 982 (1971)

4. N. Inagaki, M. Kawarabayashi, and K. Katsuura, Kogyo Kagaku Zasshi, 74, 1411 (1971)

5. P. Hofmann and F. Raschorof, Textilver., 5, 486 (1970)

6. F. A. Wodley, J. Appl. Polym. Sci, 15, 835 (1971)

7. G. A. Byrne, D. Gardiner, and F. H. Holmes, J. Appl. Chem., 16. 81 (1966)

8. K. Katsuura and N. Inagaki, Fire Retardance in Cellulose Fabrics in "Developments in Polymer Degradation", Vol. 4 (N. Gressie, Ed.), Applied Science Pub., New York, p. 259 (1982)

9. P. D. Garn and C. L. Denson, Text. Res. J., 47. 555 (1977)

10. S. Nakanishi, Kaseigaku Zasshi, 33, 76 (1982) 